\title{
Share, learn and get together: knowledge and information interactions at the XLV International Symposium of ISCEV - Hyderabad, India, 25-29 August 2007
}

\author{
Subhadra Jalali • Mineo Kondo - Raja Narayanan • \\ Chitra Kannabiran · Daphne L. McCulloch
}

Published online: 28 July 2007

(C) Springer Science+Business Media B.V. 2007

The new millennium is witness to astonishing developments in technology that have expanded our horizon in Ophthalmology. The potential for breakthrough advances to have an impact on the enigmatic and incurable retinal and neural disorders is now very real. With thousands of scientists involved in putting together various pieces of the puzzle, our newly diagnosed patients with a little-understood retinal or neural disease may feel some optimism about seeing some effective treatment within their lifetimes. Visual electrophysiology is an integral tool in the endeavour to understand and characterize the physiological basis for such disorders. Updating ourselves is a prerequisite to providing cutting-edge care to our patients.

\section{S. Jalali}

Smt Kanuri Santhamma Retina Vitreous Centre,

L.V. Prasad Eye Institute, Hyderabad, India

\section{Kondo}

Department of Ophthalmology, Nagoya University,

Nagoya, Japan

R. Narayanan $(\varangle)$

Kanuri Santhamma Retina Vitreous Service,

L.V. Prasad Eye Institute, Hyderabad, India

e-mail: narayanan@lvpei.org

C. Kannabiran

Kallam Anji Reddy Molecular Genetics Laboratory,

L.V. Prasad Eye Institute, Hyderabad, India

D. L. McCulloch

Vision Sciences, Glasgow Caledonian University, Glasgow, UK
In this rapidly expanding field involving diverse disciplines, it is not easy to follow or to understand the scope of information only from published literature or from the ubiquitous Internet. Scientific meetings provide a wonderful opportunity to interact and network with inquisitive minds. This issue of Documenta Ophthalmologica brings to you the abstracts of scientific presentations of the 45th International Symposium of the Society for Clinical Electrophysiology of Vision (ISCEV). Our society was founded in 1958 (under its former name, ISCERG) to underpin the advances in electroretinography, the first physiological technique to be widely applied in clinical ophthalmology. ISCEV now has a well-established tradition as a forum to learn, share and bring together masters and students across the whole field of clinical electrophysiology of vision in an especially congenial and informal environment. For the first time, the symposium is being held in the large Indian subcontinent. This is a golden opportunity for colleagues to participate, particularly for those who have fewer, and much more expensive, opportunities to attend international conferences of this calibre.

This year there are two special themes focused on neuro-ophthalmolgy and on retinal dystrophies as well as a special session of invited speakers focusing on the ERG in drug development.

In neuro-ophthalmology, VEPs are used primarily to probe visual function at the level of the visual cortex providing important information regarding the functional integrity of the visual system. Basic functions can be assessed by conventional flash and 
pattern VEPs. Recent advances, for example, multifocal VEPs, multi channel VEP, motion VEP and sweep VEPs have enabled us to devise more specific tests of visual function and to improve the localization and detection of VEP signals. The Symposium theme, "the evolving role of VEPs in neuro-ophthalmology", celebrates the impact of electrophsyiologic innovations on clinical and basic research of neuroophthalmology.

Over the last three decades there have been rapid advances in the understanding of the genetic basis of retinal disorders. Since the mapping of the first gene for X-linked RP in 1984, over a 100 genes for a range of retinal disorders have now been characterized. There are more genes yet to be identified since the known ones do not account for all affected families. This high degree of heterogeneity, both genetic and clinical, has rendered it very challenging to harness molecular genetic information towards benefits for patients. One aspect of defining retinal disorders from a molecular genetic standpoint is to explore whether specific gene mutations are associated with a distinctive set of manifestations of the disease-specifically, is there correlation between genotype and phenotype? Such correlations, if sufficiently specific, may serve as indicators of disease prognosis for a patient with a particular genotype. As a sensitive indicator of retinal function, electrophysiology forms an integral part of the definition of the phenotype and can provide insight into the pathophysiologic mechanism for specific genes. ERGs serve to define the extent of phenotypic variability or heterogeneity associated with a specific gene, to confirm the nature of functional differences underlying clinical heterogeneity and to provide an indication of early or mild forms of disease in mutation carriers. Some genetic mutations cause regional differences in retinal function that may be identified by multifocal ERGs. Thus, this technique offers the potential improved prognostic information when considered with specific genotypes. The Symposium theme, "the role of ocular electrophysiology in genotype-phenotype correlation" focuses on the rapid developments in genetic retinal disease.

While there may be potential for widespread genetic cures in medicine in the near future, current advances rely heavily on the unprecedented acceleration in new drug development. Each new drug requires thorough evaluation of both its safety and its efficacy.
Neuro-toxicity can be particularly difficult to detect. The retina is a highly sensitive and accessible neural tissue with exquisite neuropiles oriented to produce large and specific electrophysiologic signals, ERGs. The special session, "the ERG in pre-clinical and clinical drug development" explores the role of the ERG for drug testing in both human and animal trials.

However, the varied menu over five days goes beyond the focused themes. Looking to the future, Prof. Sumantra Chattarji's invited introductory lecture updates us on 'The Changing Brain: How visual and other behavioural experiences influence the brain." In addition, the programme includes a variety of free papers, dedicated poster sessions and a clinical cases session. A highlight of Symposium, the 2007 Adachi Award Lecture by Prof. Eberhard Zrenner, 'New Potentials for Restoring Neuroretinal Function' gives us a view into the future by reporting the cutting edge developments of retinal implants for human applications.

The Symposium deliberations are a wonderful opportunity for a wide audience who have had limited exposure to these engaging techniques: techniques that are often the tools to unlock mysteries of signs and symptoms that baffle us during patient care. The ISECV abstracts in this issue involve ophthalmologists, neurologists, neurobiologists, pharmacologists, optometrists, vision scientists, molecular geneticists, and students from related specialties. The icing on the cake is a two days pre-conference hands-on ISCEV Educational Course taught by distinguished members of the society. A bonus is the trade exhibition involving all major electrodiagnostic equipment manufacturers of the world!

The 45th ISCEV symposium is organized by $\mathrm{L} \mathrm{V}$ Prasad Eye Institute in Hyderabad, India, which brought advanced clinical electrophysiology to a country that has, on one hand, the largest number of blind people in the world and, on the other hand, the very first National Programme to control blindness! The symposium is expected to be an enriching academic and social opportunity having an international aura with traditional Indian flavour!

Editorial note: The abstracts in this issue have been edited and re-set to a standard format. We have made every effort to respect the intended meaning of the originals ands apologise for any inadvertent misinterpretation. 